\title{
Liver regeneration: immunohistochemichal study of intrinsic hepatic innervation after partial hepatectomy in rats
}

Apostolos N Kandilis ${ }^{1}$, John Koskinas ${ }^{2}$, Ioannis Vlachos ${ }^{3}$, Spyridon Skaltsas ${ }^{3}$, Despina Karandrea ${ }^{5}$, Petros Karakitsos ${ }^{6}$, Alkistis Pantopoulou ${ }^{3}$, Marina Palaiologou ${ }^{4}$, Nikolaos Nikiteas ${ }^{1}$, Dina G Tiniakos ${ }^{4,7^{*}}$ and Despina N Perrea ${ }^{3}$

\begin{abstract}
Background: We examined the intrinsic hepatic innervation after partial hepatectomy $(\mathrm{PH})$ in rats and the presence and pattern of neural sprouting in regenerating liver.

Methods: Male Wistar rats (age 9-13 weeks-w, weight 204-356 g), were submitted to two-thirds PH. Rats were sacrificed at postoperative days (d) $1,3,5,7$, at 2 and $4 \mathrm{w}$, and at 3 and 6 months $(\mathrm{m})(6-7$ animals/group, control group $n=4)$. Immunohistochemistry for the pan-neural marker protein gene product 9.5 (PGP9.5) and growth-associated protein 43 (GAP-43), a marker of regenerating nerve axons, was performed on tissue sections from the R1 lobe of the regenerating liver. Portal tracts (PTs) with immunoreactive fibers were counted in each section and computer-assisted morphometric analysis (Image Pro Plus) was used to measure nerve fiber density (number of immuno-positive nerve fibers $/ \mathrm{mm}^{2}$ (40x)).

Results: Immunoreactivity for PGP9.5 was positive in all groups. The number of PGP9.5 (+) nerve fibers decreased from $0.32+/-0.12$ (control group) to $0.18+/-0.09$ (1d post-PH group), and gradually increased reaching pre-PH levels at $6 \mathrm{~m}$ $(0.3+/-0.01)$. In contrast, immunoreactivity for GAP-43 was observed at 5d post-PH, and GAP-43 (+) PTs percentage increased thereafter with a peak at $3 \mathrm{~m}$ post-PH. GAP-43 (+) nerve fiber density increased gradually from $5 \mathrm{~d}$ $(0.05+/-0.06)$ with a peak at $3 \mathrm{~m}$ post-PH $(0.21+/-0.027)$. At $6 \mathrm{~m}$ post-PH, immunoreactivity for GAP-43 was not detectable.

Conclusions: Following PH in rats: 1) nerve fiber density in portal tracts decreases temporarily, and 2) neural sprouting in the regenerating liver lobes starts at $5 \mathrm{~d}$, reaches peak levels at $3 \mathrm{~m}$ and disappears at $6 \mathrm{~m}$ post-PH, indicating that the increase in hepatic mass after $\mathrm{PH}$ provides an adequate stimulus for the sprouting process.
\end{abstract}

\section{Background}

The extraordinary ability of the liver to regenerate following injury or resection is a property that was recognized by ancient Greeks in the well-known myth of Prometheus and the less known myth of Tityus [1]. Liver regeneration is a very complex process involving the activation and interaction of multiple cytokines and growth factors that regulate cell growth and proliferation. During the regenerative process after partial hepatectomy $(\mathrm{PH})$, liver cells

\footnotetext{
*Correspondence: dina.tiniakos@ncl.ac.uk

${ }^{4}$ Laboratory of Histology and Embryology, Medical School, National and Kapodistrian University of Athens, Athens, Greece

${ }^{7}$ Institute of Cellular Medicine, Faculty of Medical Sciences, Newcastle University, William Leech Bldg, 4th Floor, Room M4.143, Framlington Place, Newcastle upon Tyne NE2 4HH, UK

Full list of author information is available at the end of the article
}

continue to function, while undergo mitosis in order to re-establish the organ's mass. In the rat, restoration of hepatic mass is completed in 5-7 days following $\mathrm{PH}$, whereas liver architecture in terms of sinusoidal ultrastructure is restored in 10-14 days [2-6]. On the other hand, little is known about the nerves in the regenerating liver. Ungvary et al. were the first to study the effect of $\mathrm{PH}$ on the monoaminergic nerves of the liver [7]. Pietroletti et al. studied hepatic innervation in a rat model after $\mathrm{PH}$ with the use of immmunohistochemistry [8], and Carobi examined the possibility of neural sprouting in the regenerating rat liver following $\mathrm{PH}$ [9].

Hepatic re-innervation after experimental orthotopic liver transplantation (OLT) has been studied in the past 
using immunohistochemistry with antibodies to protein gene product 9.5 (PGP 9.5) and growth-associated protein43 (GAP-43) in rat models [10,11]. PGP9.5 belongs to the ubiquitin carboxy-terminal hydrolases [12]. It is expressed in neurons and neuroendocrine cells of vertebrates, and is present within the axoplasm of both peripheral and central nerve fibers, thus rendering it an excellent marker for nerve axons [13]. An important drawback of PGP9.5 is the lack of discrimination between normal and regenerating axons [11]. GAP-43 is a protein exclusively expressed in the nervous system. Its expression is related to axonal growth during neuronal development and regeneration and, therefore, GAP-43 is a useful marker for developing or regenerating nerve axons [14-16].

The present study was conducted to examine alterations of the intrinsic hepatic innervation at several time points following $\mathrm{PH}$ in rats, using the neuronal markers PGP9.5 and GAP-43. In addition, the possible role of the increase in hepatic mass after $\mathrm{PH}$ as an adequate stimulus for neural sprouting was evaluated.

\section{Methods}

Fifty six male Wistar rats, with a mean weight of $283 \mathrm{~g}$, were purchased by the National Centre of Scientific Research "DEMOKRITOS" (Athens, Greece). The animals had free access to water and food, and $12 \mathrm{~h}$ before surgery they were deprived only of food. Four/56 animals were randomly chosen to consist the control group and were not submitted to an operation, while the remaining 52 were submitted to two-thirds partial hepatectomy $(\mathrm{PH})$, according to Higgins and Anderson [17], under ether anesthesia. Following $\mathrm{PH}$, rats were sacrificed at postoperative days (PODs) $1,3,5,7$, at 2 and 4 weeks, and at 3, and 6 months post-PH. Each group consisted of 6 or 7 animals. The liver was then removed en block and crosscut, 3-mm-thick tissue specimens were obtained from the anterior sub-lobe (R1) of the rat liver right lobe [18].

The study was approved by the Ethics Committee of the National and Kapodistrian University of Athens and was carried out according to the strict regulations concerning animal care set by this committee.

\section{Immunohistochemistry}

Tissue blocks consisting of two or three crosscut specimens from the R1 lobe were fixed for $24 \mathrm{~h}$ in $10 \%$ neutral formalin and then were routinely paraffin-embedded. Five$\mu \mathrm{m}$-thick serial sections were cut from each block and were mounted onto poly-L lysine-coated slides. One section/case was stained with haematoxylin and eosin stain for conventional histological evaluation. Immunochemistry was performed manually using the Novo Castra Novolink
Polymer Detection Kit, according to the manufacturer's instructions. After deparaffinization and rehydration of the sections in a series of graded ethanols, endogenous peroxidase was quenched with $1 \% \mathrm{H} 2 \mathrm{O} 2$ in methanol for $30 \mathrm{mi}-$ nutes (min). After three 4-min treatments in $0.001 \mathrm{M}$ citrate buffer $(\mathrm{pH}=6)$ in a microwave oven $(800 \mathrm{~W})$ for antigen unmasking, sections were incubated overnight in $4^{\circ} \mathrm{C}$ with the primary antibodies specific for PGP9.5 (Novocastra Laboratories, UK, mouse CLONE 1DA1, diluted 1:100) and GAP-43 (Zymed Laboratories, USA, mouse CLONE 7B10, diluted 1:150), followed by incubation with the secondary antibodies coupled with polymer-horseradish peroxidase (Novolink Polymer) for $25 \mathrm{~min}$ at room temperature. All steps were carried out in a $0.25 \mathrm{M}$ phosphate buffer saline (PBS; $\mathrm{pH}=7.4$ ) at $25^{\circ} \mathrm{C}$. After being rinsed with tap water, sections were counterstained with hematoxylin for $15 \mathrm{sec}$, dehydrated in graded ethanols, cleared in xylene, mounted with DPX (BDH, England), and coverslipped. A negative control, in which the primary antibody was omitted, was used in every case. Sections of the central nervous system of a rat embryo were used as positive controls for both neuronal markers, as previously reported [11].

\section{Computer-assisted morphometric analysis of immunoreactive nerve fibers}

Morphometric variables were determined by image analysis, using an IBM computer (IBM Computer, Inc.; Armonk, NY). Images were captured with a Leica DM LB microscope coupled to a Sony digital camera (DFW-X700) and digitalized with Path-Sight version 4.3 (Medical Solutions plc.; Nottingham, UK).

We examined a mean of 7 portal tracts (PTs) per section (range 5-14). Each section was obtained from the R1 lobe of the liver rat, $0.5-1 \mathrm{~cm}$ from the hepatic hilus. Hot spot areas, i.e. PTs with positive immunostaining were further analyzed using Image Pro Plus (Media Cybernetics, Inc.; Silver Spring, MD). The size of the PTs was not always small enough to fit entirely into one single image (photograph) by the digital camera; therefore serial images were taken, each of them consisting of a variable number of immunostained nerve fibers. The computerized system measured the number of nerve fibers per $\mathrm{mm}^{2}$ in the hot spot areas of $40 \mathrm{x}$ objective/section/region [19].

\section{Statistical analysis}

Data are expressed as mean \pm 1 standard deviation (S.D.). The normality of the distributions was assessed with Kolmogorov-Smirnov test and graphical methods. Comparisons between more than two groups were performed with Analysis of Variance (ANOVA). In cases of multiple hypothesis testing, Benjamini \& Hochberg's False Discovery Rate (FDR) was utilized in order to assess 
between-group differences, as well as to control familywise error to $<0.05$. The GAP-43 and PGP9.5 models were implemented using cubic B-Splines. All tests were twosided. Differences were considered as statistically significant if the null hypothesis could be rejected with $>95 \%$ confidence $(\mathrm{p}<0.05)$.

\section{Results}

Immunoreactivity for PGP9.5

PGP9.5-positive nerve fibers were detected during the entire study period, with alterations in their density (number of nerve fibers $/ \mathrm{mm}^{2}$ ) and number of positive portal tracts (PTs) (Figure 1A). In all instances, the
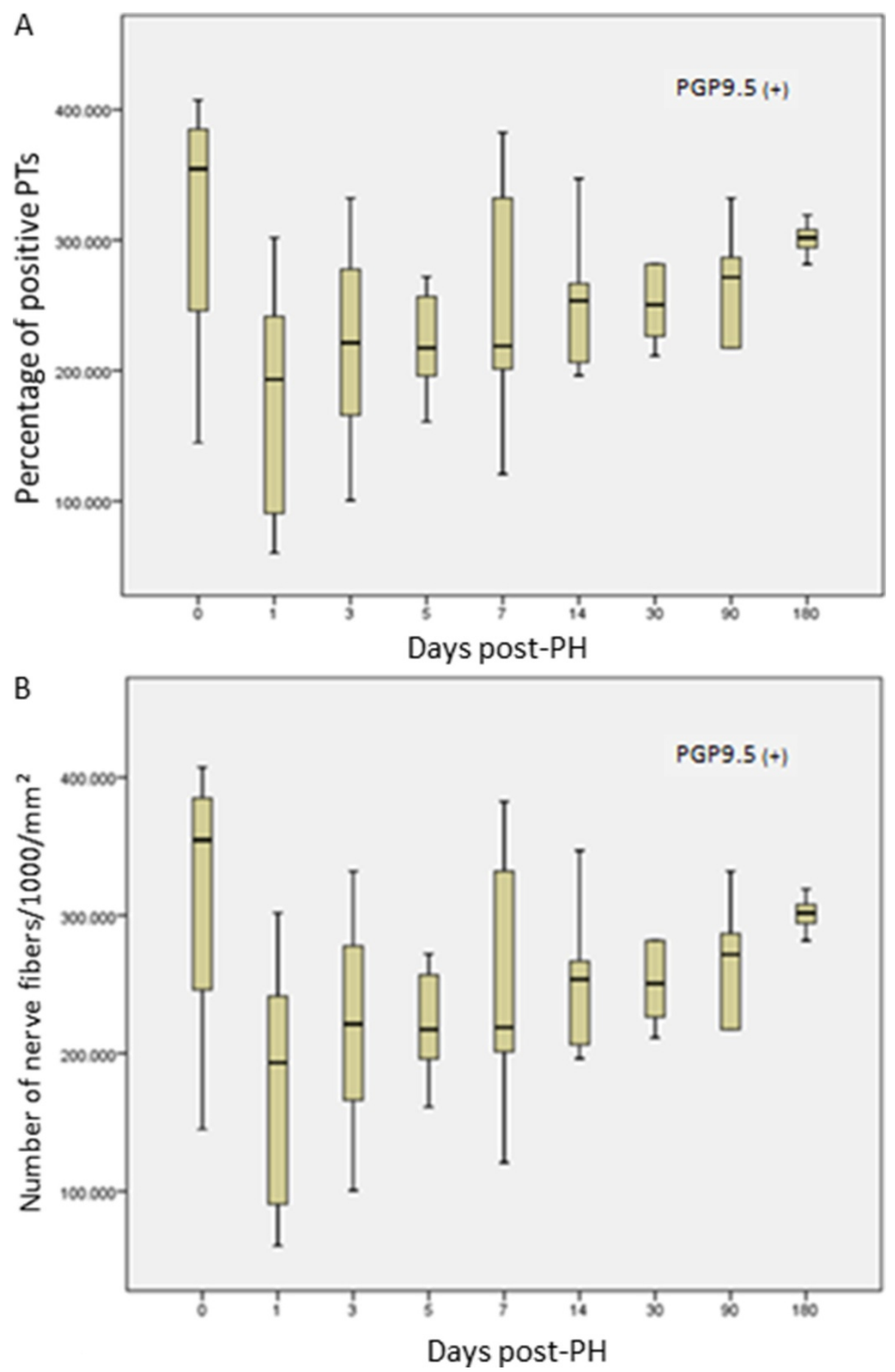

Figure 1 Change in immunoreactivity for PGP9.5 following PH. A. Fluctuation of percentage of PGP 9.5 (+) PTs, B. Fluctuation of density of PGP 9.5 (+) nerve fibers $/ \mathrm{mm}^{2}$. 
immunohistochemical reaction, when positive, was localised exclusively in PTs (Figure 2). At POD 1, nerve fiber density was substantially decreased at $0.180(+/-0.091)$ in comparison to the control group $(0.315+/-0.116)(\mathrm{p}=$ $0.0507)$. Thereafter, density gradually increased to 0.220 $(+/-0.084)$ at POD $3,0.245(+/-0.995)$ at 1 week, 0.266 $(+/-0.044)$ at 3 months, and, lastly, reached the prehepatectomy level at 6 months post-PH $(0.30+/-0.013)$ (Figure 1B).

Furthermore, at POD 1, the percentage of PGP9.5positive PTs was decreased to $46.42 \%(44.67+/-12.98)$ from $58.62 \%(59.00+/-7.26)$ in the control group ( $\mathrm{p}>$ $0.05)$ and gradually increased to $56.33 \%$ (56.14 +/7.358) at 6 months post-PH.

\section{Immunoreactivity for GAP-43}

GAP-43 immunoreactivity was absent in the control group $(0 / 4)$ and at POD 1 and 3 . GAP-43 positive nerve fibers were firstly identified at POD 5 in PTs of some specimens $(3 / 7)$, whereas no immunoreactivity was evident in the control group (0/4) and at PODs $1(0 / 6)$ and $3(0 / 7)$. Thereafter, GAP-43 was expressed in all specimens at 1 (6/6) and $3(6 / 6)$ months post-PH. At 6 months post- PH, no GAP-43-positive nerves (0/7) were detected (Figure 3). Similarly to PGP9.5 immunostaining results, GAP-43specific immunopositivity was localised exclusively at PTs (Figure 4). At POD 5, when GAP-43-immunopositivity was first detected, the density of GAP-43-positive fibers was $0.047(+/-0.060)$. It gradually increased to $0.095(+/-$

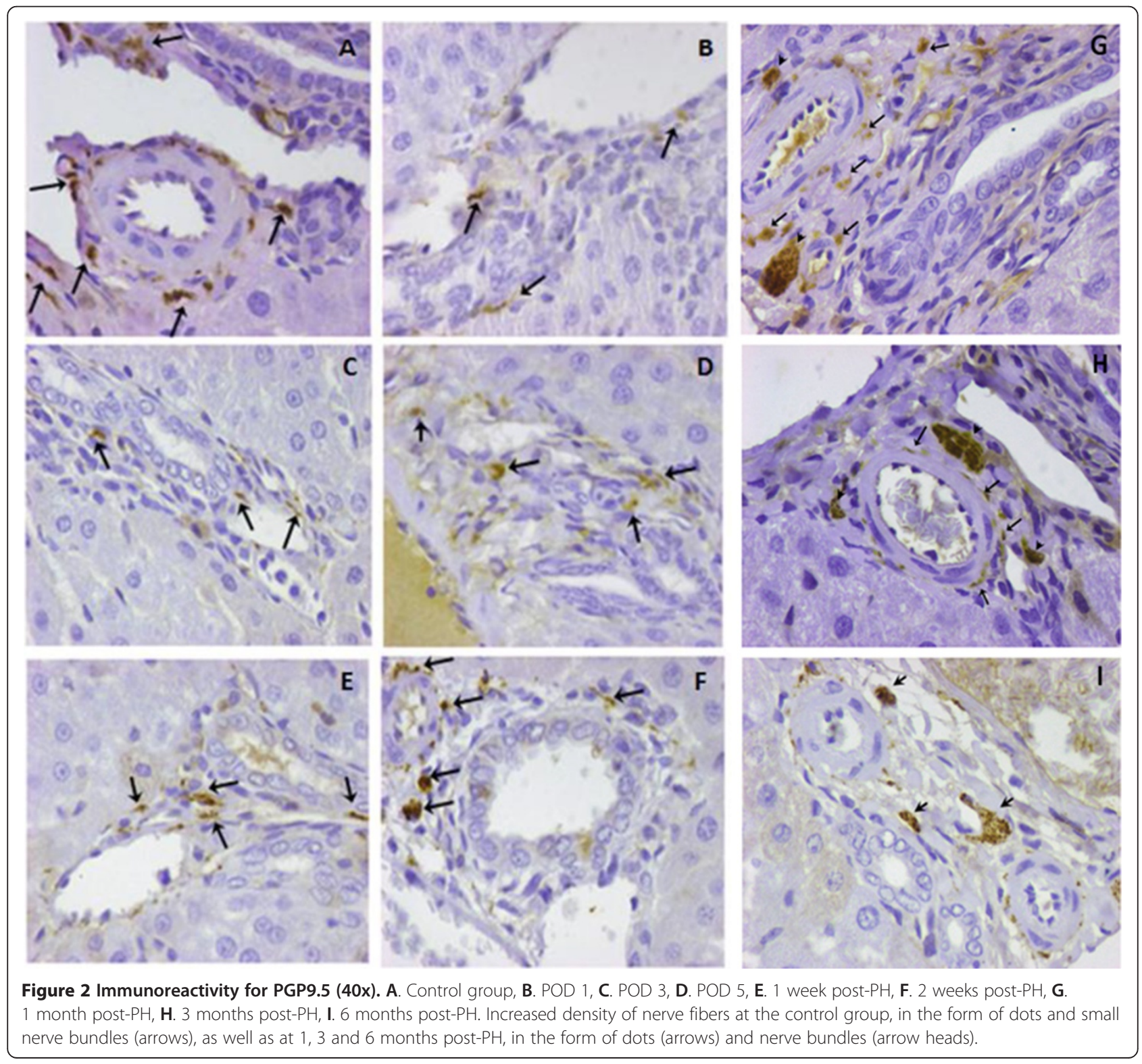


$0.081)$ at 1 week, $0.140(+/-0.087)$ at 2 weeks, $0.169(+/-$ $0.024)$ at 1 month, to reach a peak at 3 months $(0.210+/-$ 0.027 ) post-PH (Figure $3 \mathrm{~A}$ ). The difference in density between the groups at POD 5 and 1 week post-PH was not statistically significant $(p>0.05)$, whereas differences between POD 5 and 2 weeks, 1 month and 3 months post-PH were statistically significant at a level of $\mathrm{p}<0.01$, $\mathrm{p}<0.001$, and $\mathrm{p}<0.0001$, respectively. No intraparenchymal GAP-43-positive fibers were observed in either the control or the experimental group.
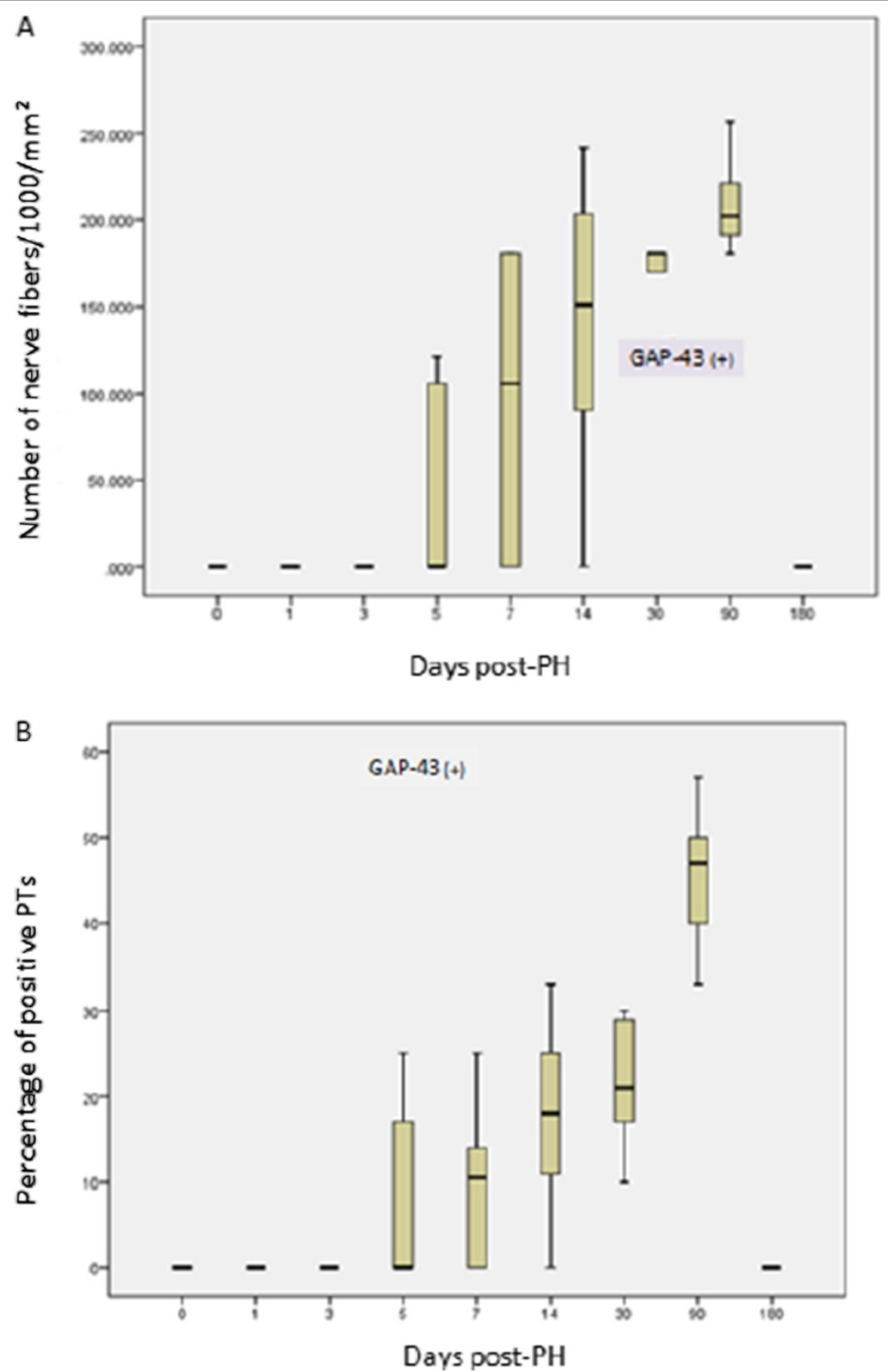

Figure 3 Change in immunoreactivity for GAP-43 following PH. A. Fluctuation of density of GAP-43 (+) nerve fibers $/ \mathrm{mm}^{2}$, B. Fluctuation of percentage of GAP-43 (+) PTs. 


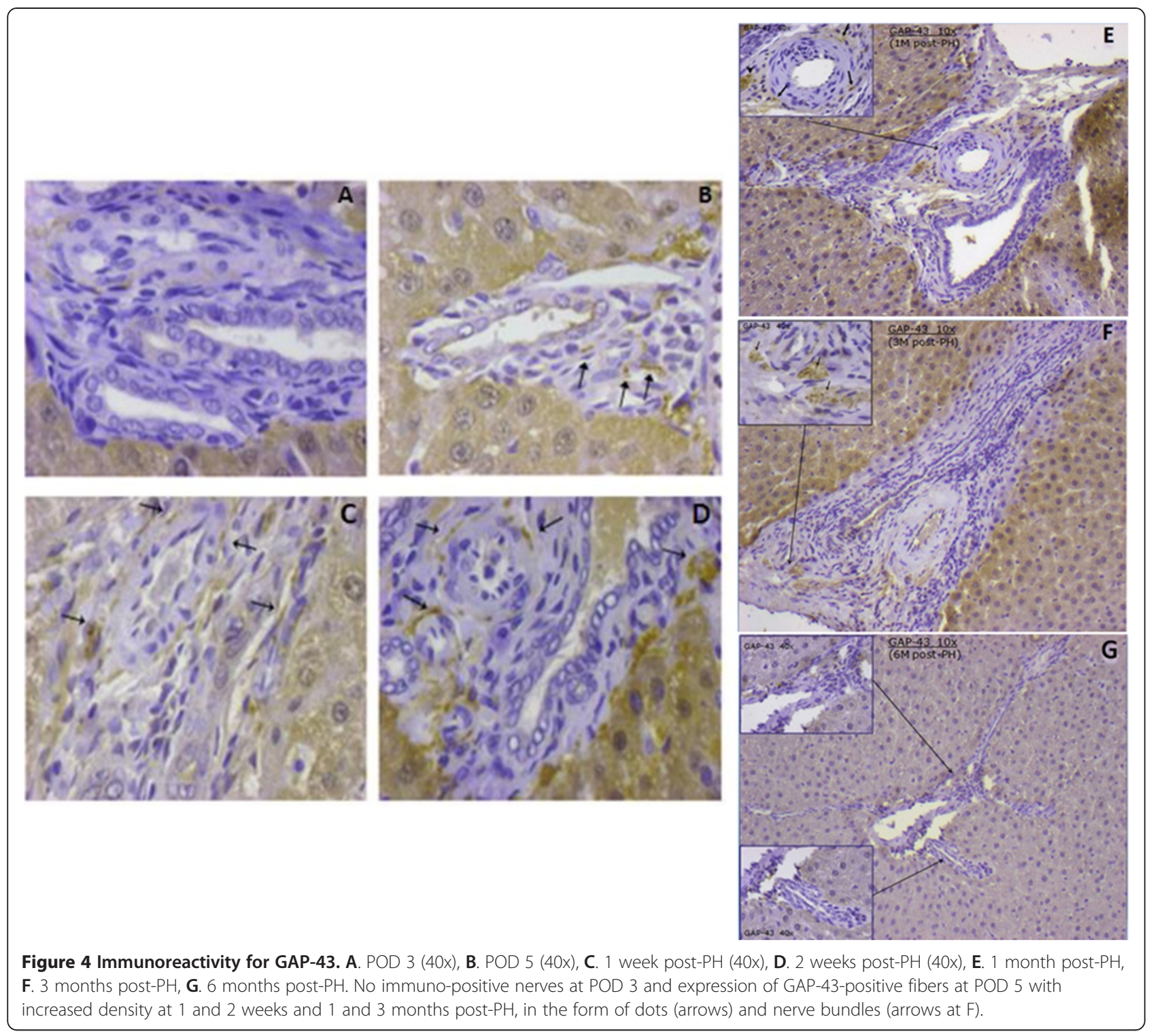

At POD 5, the mean percentage of GAP-43-positive PTs was $8.43 \%(+/-11.22)$, then increased steadily to $10.0 \%$ $(+/-9.40)$ at 1 week, $17.57 \%(+/-11,15)$ at 2 weeks, $21.33 \%(+/-7.53)$ at 1 month to reach the highest level at 3 months $(45.67 \%+/-8.50)$ post-PH (Figure $3 \mathrm{~B})$.

\section{Discussion}

We have shown that during liver regeneration after $\mathrm{PH}$, the number of PGP9.5-positive nerve fibers was decreased at POD 1 and subsequently gradually recovered reaching the levels of the control group at 6 months. Furthermore, PGP9.5-positive axons were present exclusively in PTs of every specimen at all time points after $\mathrm{PH}$, whereas the expression of GAP-43 became positive in PTs at POD 5, increased to a peak at 3 months, and disappeared at 6 months post-PH.
The exclusive co-expression of PGP9.5- and GAP-43positive nerve fibers in portal tracts is related to the specific nerve distribution in the rat liver. Intrahepatic nerve distribution is highly species-dependant and the most prominent feature in the rat is the lack of intralobular innervation and mere presence of nerve fibers in PTs [20]. In contrast, human liver shows high nerve density in the lobules, while nerve fibers are also detected in PTs [21].

The transient reduction of PGP9.5 positive axons early after PH is in keeping with the results of Ungvary et al. and can be attributed to the prevalence of liver cell proliferation in order to restore the organ's mass, proceeding in a faster manner than the innervation of the regenerating liver [7]. After examining the monoaminergic nerve fibers, Ungvary et al. found no innervation at 
the periphery of the regenerating lobules at POD 1, and restoration of nerve density at 6 weeks post-PH. Such an alteration was not encountered at the hepatic hilus, where, on the contrary, increased innervation was observed at POD 7 [7]. Hyperinnervation was also noted by Pietroletti et al. in their immunohistochemical study of the neuronal markers neuron specific enolase, neurofilaments and protein $\mathrm{S}-100$ in rats after $\mathrm{PH}$ [8]. In addition to the importance of the dual blood supply for the optimal regenerative response, they detected more intense immunoreactivity and more nerve fibers in the group of hepatectomised rats at POD 10 compared to the control group. This difference was attributed to the higher neuronal metabolic rate during regeneration. The limitation of this study was that it was conducted at one time point after PH only (POD 10) thus restricting the significance of the findings [8].

The expression of GAP-43, a neuronal marker expressed in developing or regenerating axons, after $\mathrm{PH}$ is indicative of the presence of neural sprouting in the regenerating liver and indicates that the compensatory growth of the hepatic mass is the triggering stimulant. The timing of GAP-43 expression following PH is different than that of the liver cellular components or angiogenic events during the regenerative response. Hepatic mass and architecture are restored by POD 14 [3], whereas GAP-43 immnunoreactivty showed delayed onset at POD 5 and termination between 3 and 6 months post-PH. Ungvary et al. in 1974 were the first to study the effect of $\mathrm{PH}$ on liver innervation and concluded that nerve regeneration takes place in the regenerating organ, with the new neural elements growing out from intact nerves [7]. Neural sprouting was examined again in 1990, with the method of retrograde transport of horseradish peroxidase (HRP) for the study of vagal afferent neurons after $\mathrm{PH}$ in rats. At 3 weeks post-PH, no neural sprouting was evident in the regenerating liver [9].

The expression of PGP9.5 and GAP-43 was studied after OLT in rats and showed that hepatic reinnervation occurs in the transplanted liver at the porta hepatis $[10,11]$. The timing pattern of immunoreactivity has certain similarities to our study, with PGP9.5-positive axons present during the entire study period, whereas GAP-43positive axons were present in some specimens at POD 3 and sustained until 3 months post-PH. No innervation was noted in the liver parenchyma $[10,11]$. The human transplanted liver, although inevitably denervated, maintains its function [22] and results from studies regarding hepatic reinnervation have been controversial. Both Dhillon et al. [23] and Boon et al. [24] have reported possible hepatic reinnervation after liver transplantation, while Kjaer et al. showed no evidence of liver sympathetic nerve fiber regeneration [25]. The latter finding is supported by a later study by May et al. who analysed sympathetic activation induced by water drinking on patients subjected either to OLT or to kidney transplantation and found reduced plasma norepinephrine levels in the OLT group after drinking water, implying impaired response to the stimulant due to liver graft denervation [26].

Hepatic nerve regeneration has been studied using GAP-43 immunohistochemistry in dogs following surgical denervation. GAP-43 positive nerve fibers were seen at 1 month post-denervation and absence of expression at 1 week and 3 months after the procedure was then observed [27].

Our study is the first to examine the expression of neuronal markers PGP9.5 and GAP-43 at several $(n=8)$ time points post-PH. Most of the related literature to date, with exception studies after OLT, originate from studies on liver innervation based on few time points only following either $\mathrm{PH}$ or surgical denervation. However, our study has certain limitations, namely the fact that it is based only in immunochemistry without the use of electron microscope and the porta hepatis was not examined. Furthermore, although many time points were used, it is difficult to reach to the general conclusion that the peak of hepatic reinnervation occurs at 3 months, since the interval until 6 months post-PH, when there is no expression of GAP-43, is quite long.

\section{Conclusion}

Our results have shown that during liver regeneration after $\mathrm{PH}$, the process of neural sprouting starts at POD 5 and terminates between 3 and 6 months post-PH. Taking into account the absence of intralobular innervation in the rat and the exclusive expression of both PGP9.5 and GAP-43 in the PTs, neural sprouting is considered to take place in the PTs of the regenerating liver. This finding indicates that possibly new PTs are created to accommodate the elongating nerve fibers. New PTs could further lead to new lobules as part of the regenerating response [28]. The question of new lobules or hyperplasia of existing ones during liver regeneration still remains and was addressed in the past by methods of measuring the size and number of liver lobules with conflicting results [29-33]. Further studies are needed to examine if GAP-43, a neuronal marker exclusively expressed in regenerating nerve axons and in PTs in the rat, could be used as a marker of new PTs in the regenerating rat liver, in order to accommodate the elongating nerve fibers, and further as a marker of new liver lobules.

\section{Competing interests}

The authors declare that they have no competing interests.

\section{Authors' contributions}

ANK performed the conception, design and most of the experimental part of the study and wrote the manuscript. JK performed the conception and design of the study, critically revised and helped to draft the manuscript. IV 
perfomed the statistical analysis and participated in the design of the study. SS carried out most of the experimental part and participated in the design of the study. DK carried out the immunohistochemichal analysis and participated in the design of the study. PK and AP assisted with the experimental part and participated in the design of the study. MP assisted with immunohistochemistry and participated in the design of the study. NN participated in the design of the study and helped to draft the manuscript. DGT performed the conception and design of the study, assisted with immunohistochemistry, critically revised and helped to draft the manuscript. DNP performed the design of the study, assisted with the experimental part and helped to draft the manuscript. All authors read and approved the final manuscript.

\section{Authors' information}

Dina G Tiniakos and Despina N Perrea share senior authorship.

\section{Acknowledgements}

We thank Dr Serafim Tsoukos for his technical assistance using the Image Pro Plus. We would also like to thank the staff of both the Laboratory for Experimental Surgery and Surgical Research "N. S. Christeas" and the Laboratory of Histology and Embryology for their cooperation in the conduction of the experimental protocol.

\section{Author details}

${ }^{1}$ Second Propedeutic Department of Surgery, Medical School, University of Athens, General Hospital "Laiko", Athens, Greece. ${ }^{2}$ Second Department of Medicine, Medical School, University of Athens, "Hippokration" Hospital, Athens, Greece. ${ }^{3}$ Laboratory for Experimental Surgery and Surgical Research "N. S. Christeas", Medical School, University of Athens, Athens, Greece. ${ }^{4}$ Laboratory of Histology and Embryology, Medical School, National and Kapodistrian University of Athens, Athens, Greece. ${ }^{5}$ Department of Pathology, Aretaieion Hospital, Athens, Greece. ${ }^{6}$ Department of Cytopathology, University General Hospital "ATTIKON", School of Medicine, National and Kapodistrian University of Athens, Athens, Greece. ${ }^{7}$ Institute of Cellular Medicine, Faculty of Medical Sciences, Newcastle University, William Leech Bldg, 4th Floor, Room M4.143, Framlington Place, Newcastle upon Tyne NE2 $4 \mathrm{HH}, \mathrm{UK}$.

Received: 20 August 2014 Accepted: 11 November 2014 Published online: 25 November 2014

\section{References}

1. Tiniakos DG, Kandilis A, Geller SA: Tityus: a forgotten myth of liver regeneration. J Hepatol 2010, 53(2):357-361.

2. Michalopoulos GK: Liver regeneration. J Cell Physiol 2007, 213(2):286-300.

3. Michalopoulos GK, DeFrances MC: Liver regeneration. Science 1997, 276(5309):60-66.

4. Taub R: Liver regeneration: from myth to mechanism. Nat Rev Mol Cell Biol 2004, 5(10):836-847.

5. Fausto N, Campbell JS, Riehle KJ: Liver regeneration. Hepatology 2006, 43(2 Suppl 1):S45-S53.

6. Martinez-Hernandez A, Amenta PS: The extracellular matrix in hepatic regeneration. FASEB J 1995, 9(14):1401-1410.

7. Ungvary G, Donath T, Naszaly SA: Regeneration of the monoaminergic nerves in the liver after partial hepatectomy. Acta Morphol Acad Sci Hung 1974, 22(2):177-186.

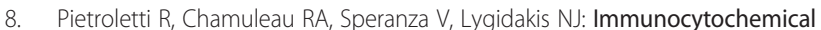
study of the hepatic innervation in the rat after partial hepatectomy. Histochem J 1987, 19(6-7):327-332.

9. Carobi C: Vagal afferent innervation in regenerated rat liver. Experientia 1990, 46(1):50-53.

10. Sakamoto I, Takahashi T, Kakita A, Hayashi I, Majima M, Yamashina S: Experimental study on hepatic reinnervation after orthotopic liver transplantation in rats. J Hepatol 2002, 37(6):814-823.

11. Takahashi T, Kakita A, Sakamoto I, Takahashi Y, Hayashi K, Tadokoro F, Yamashina S: Immunohistochemical and electron microscopic study of extrinsic hepatic reinnervation following orthotopic liver transplantation in rats. Liver 2001, 21(5):300-308.

12. Wilkinson KD, Lee KM, Deshpande S, Duerksen-Hughes P, Boss JM, Pohl J: The neuron-specific protein PGP 9.5 is a ubiquitin carboxyl-terminal hydrolase. Science 1989, 246(4930):670-673.
13. Wilson PO, Barber PC, Hamid QA, Power BF, Dhillon AP, Rode J, Day IN, Thompson RJ, Polak JM: The immunolocalization of protein gene product 9.5 using rabbit polyclonal and mouse monoclonal antibodies. $\mathrm{Br} J$ Exp Pathol 1988, 69(1):91-104.

14. Van Hooff CO, De Graan PN, Oestreicher AB, Gispen WH: B-50 phosphorylation and polyphosphoinositide metabolism in nerve growth cone membranes. J Neurosci 1988, 8(5):1789-1795.

15. Verge VM, Tetzlaff W, Richardson PM, Bisby MA: Correlation between GAP43 and nerve growth factor receptors in rat sensory neurons. J Neurosci 1990, 10(3):926-934.

16. Widmer F, Caroni P: Phosphorylation-site mutagenesis of the growthassociated protein GAP-43 modulates its effects on cell spreading and morphology. J Cell Biol 1993, 120(2):503-512.

17. Higgins GMAR: Experimental pathology of the Liver. I. restoration of the liver of the white rat following partial surgical removal. Arch Pathol 1931 $12: 186-202$

18. Kogure K, Ishizaki M, Nemoto M, Kuwano H, Makuuchi M: A comparative study of the anatomy of rat and human livers. $J$ Hepatobiliary Pancreat Surg 1999, 6(2):171-175.

19. Anagnostou VK, Doussis-Anagnostopoulou I, Tiniakos DG, Karandrea D, Agapitos E, Karakitsos P, Kittas C: Ontogeny of intrinsic innervation in the human thymus and spleen. J Histochem Cytochem 2007, 55(8):813-820.

20. Uyama N, Geerts A, Reynaert H: Neural connections between the hypothalamus and the liver. Anat Rec A: Discov Mol Cell Evol Biol 2004, 280(1):808-820.

21. Bioulac-Sage $P$, Lafon ME, Saric J, Balabaud C: Nerves and perisinusoidal cells in human liver. J Hepatol 1990, 10(1):105-112.

22. Colle I, Van Vlierberghe H, Troisi R, De Hemptinne B: Transplanted liver: consequences of denervation for liver functions. Anat Rec A: Discov Mol Cell Evol Biol 2004, 280(1):924-931.

23. Dhillon AP, Sankey EA, Wang JH, Wightman AK, Mathur S, Burroughs AK, Scheuer PJ: Immunohistochemical studies on the innervation of human transplanted liver. J Pathol Bacteriol 1992, 167(2):211-216.

24. Boon AP, Hubscher SG, Lee JA, Hines JE, Burt AD: Hepatic reinnervation following orthotopic liver transplantation in man. J Pathol Bacteriol 1992, 167(2):217-222.

25. Kjaer M, Jurlander J, Keiding $\mathrm{S}$, Galbo H, Kirkegaard P, Hage E: No reinnervation of hepatic sympathetic nerves after liver transplantation in human subjects. J Hepatol 1994, 20(1):97-100.

26. May M, Gueler F, Barg-Hock H, Heiringhoff KH, Engeli S, Heusser K, Diedrich A, Brandt A, Strassburg CP, Tank J, Sweep FC, Jordan J: Liver afferents contribute to water drinking-induced sympathetic activation in human subjects: a clinical trial. PLOS One 2011, 6(10):e25898.

27. Ito Y, Takahashi T, Tadokoro F, Hayashi K, lino Z, Sato K, Akira K: Regeneration of the hepatic nerves following surgical denervation of the liver in dogs. Liver 1998, 18(1):20-26.

28. Papp V, Dezso K, Laszlo V, Nagy P, Paku S: Architectural changes during regenerative and ontogenic liver growth in the rat. Liver Transp/ 2009, 15(2):177-183.

29. Simpson GE, Finckh ES: The pattern of regeneration of rat liver after repeated partial hepatectomies. J Pathol Bacteriol 1963, 86:361-370.

30. lashina IN: Formation of hepatic lobules in the regenerating liver. Biull Eksp Biol Med 1970, 70(10):95-98.

31. Sidorova VF: On the structure of the regenerating liver in a rat. Biull Eksp Biol Med 1959, 48:99-104.

32. latropoulos $\mathrm{MJ}$ : Cytoarchitecture of rat liver during compensatory growth. Anat Rec 1971, 169(3):509-514.

33. Wagenaar GT, Chamuleau RA, Pool CW, De Haan JG, Maas MA, Korfage HA, Lamers WH: Distribution and activity of glutamine synthase and carbamoylphosphate synthase upon enlargement of the liver lobule by repeated partial hepatectomies. J Hepatol 1993, 17(3):397-407.

doi:10.1186/s12876-014-0202-1

Cite this article as: Kandilis et al.: Liver regeneration:

immunohistochemichal study of intrinsic hepatic innervation after partial hepatectomy in rats. BMC Gastroenterology 2014 14:202. 